\title{
Field evaluation of WHO-MDT of fixed duration, at ALERT, Ethiopia: the AMFES project-II. Reaction and neuritis during and after MDT in PB and MB leprosy patients
}

\author{
A. J. DE RIJK, ${ }^{*} \ddagger$ SHIBRU GABRE, $*$ P. BYASS $\dagger \&$ \\ THEODROES BERHANU* \\ * All Africa Leprosy Rehabilitation and Training Centre ( ALERT), \\ PO Box 165, Addis Ababa, Ethiopia; †Dept. of Public Health \\ Medicine and Epidemiology, University of Nottingham, Queen's \\ Medical Centre, Nottingham, UK
}

\section{Accepted for publication 9 March 1994}

\begin{abstract}
Summary For a cohort of 286 leprosy patients the incidence rates and clinical manifestations of leprosy reactions during treatment and surveillance are described. Currently, individual patients had been observed for up to 4 years. It is intended that surveillance within this project should continue for up to 5 years after treatment. Of $128 \mathrm{~PB}$ patients, observed for 267 person-years (mean $2 \cdot 1) 27$ had 35 episodes of reaction, corresponding to an overall incidence rate of 131 events per 1000 person-years-at-risk (pyar).

Of $158 \mathrm{MB}$ patients observed for 402 person years (mean 2.5), 64 had 114 reactions, with an overall incidence of 284 events per 1000 pyar. For both PB and MB patients, incidence rates during treatment and post-MDT surveillance were similar. For PB patients, pre-existing physical impairment at the start of MDT was a significant risk factor for the occurrence of subsequent events, but this was not found in MB patients.
\end{abstract}

\section{Introduction}

The purpose and design of ALERT's MDT Field Evaluation Studies (the AMFES project) have been described in Part I, giving data on enrolment, completion of treatment and case holding for 286 new leprosy patients observed for 15-51 months, during and after the MDT course. In this second part we give our findings regarding the occurrence of leprosy reactions, neuritis and nerve dysfunction in this cohort of patients during the 4.25 year period from 1 April 1988 to 30 June 1992.

‡Correspondence: c/o SNV, Netherlands Development Organisation, PO Box 40675, Addis Ababa, Ethiopia. 


\section{Methods}

At every 4-weekly drug collection, each patient is briefly examined by the clinic's health assistant (HA), who asks for any complaints, particularly regarding eyes, hands or feet, and inspects hands and feet for wounds, tests for changes in muscle strength of eyelids, hands and feet by a standard set of voluntary muscle tests (VMT) and in the sensory capacity of eyes, palms and soles. Sensory testing (ST) of palms and soles was, until July 1989, done with the tip of a ballpoint pen; thereafter a nylon filament applying a much more standardized pressure stimulus of $10 \mathrm{~g}$ was used at a standard series of test points. For the eyes, normal blinking was taken as circumstantial evidence of normal sensation. Only in the absence of normal eye blinking was the sensation of the cornea tested by touching the limbus with a fine wisp of cottonwool.

The HA also palpates 4 peripheral nerve trunks (of the ulnar, median, lateral popliteal and tibialis posterior nerves), on both sides, for tenderness. All findings of this examination are recorded on the individual 'Patient Routine Care Form', which is kept by the health assistant. The procedures of this 'VMT and ST', and its recording, done at every 4-weekly treatment session, are exactly the same in this study as in the routine leprosy control programme. In case of any complaint which may point towards a leprosy reaction, and for each finding of nerve tenderness, or of any deterioration in nerve function, the health assistant has to present the patient to the leprosy control supervisor (LCS) for assesment of a possible 'event' (see below).

During treatment, all patients are also periodically examined by the LCS. For PB patients this is done at the 6th treatment round, before the patient is reminded that next month the treatment course will be completed. MB patients have a full review by the LCS at treatment rounds 7, 13, 19 and 25 or 26 . At each of these review examinations, the LCS assesses the skin lesions, takes skin smears from 4 sites, does all routine nerve function assessments and records the findings on the Patient Record Card.

During the 5-year surveillance, periodical reviews are done by the LCS at 3 months, 6 months, and thereaf ter every 6 months. Patients are, however, also explicitly advised to come at any time when they notice a problem of reaction or nerve function loss. Caseholding procedures during treatment and during surveillance, and results achieved, have been given in Part I, together with methodological details. ${ }^{1}$

\section{EVENTS}

In AMFES, an event is defined as noticeable signs or symptoms associated with leprosy reaction, new nerve dysfunction or a relapse of the disease.

In this field service-based study, we distinguish between only 2 types of leprosy reactions: reversal reaction (RR) or type 1 reaction, occurring mainly in $\mathrm{BT}$ and $\mathrm{BL}$ patients and associated with cell-mediated hypersensitivity. This category may include reactions which some leprologists may prefer to call 'downgrading reactions', erythema nodosum leprosum (ENL), or type 2 reaction, occurring in $\mathrm{LL}$ and $\mathrm{BL}$ patients and associated with humoral (antibody mediated) responses to antigens of Mycobacterium leprae, with immune complexes.

A further distinction is made between mild and severe forms for both RR and ENL reactions. Criteria for assessment of reactions are the same as those used in our routine leprosy control programme, given in ALERT's Manual ${ }^{2}$ and summarized in Figure 1. 
Signs of reversal reactions, skin component only, ' $R R$ ':

Mild skin involvement

— skin lesions become red and raised

- appearance of new lesions, and/or increase in size of pre-existing lesions

- paraesthesia or hypersensitivity in pre-existing skin lesions

Severe skin involvement

- skin lesions become red, raised, very tender and ulcerate

- skin reaction in a patch overlying a major nerve trunk or the eye (here the skin involvement as such may not be severe, but in the instructions for treatment it is grouped with 'severe' because of the high risk of nerve involvement

- marked oedema of hands or feet

Signs of erythema nodosum leprosum ' $E N L$ ':

Mild ENL reaction

- appearance in the skin of red and painf ul nodules which may disappear af ter a few days, while other similar nodules come up; without ulceration of skin

-nerve involvement: thickening of nerves and/or paraesthesia, without tenderness on palpation or loss of function: mild neuritis

- mild fever and/or malaise

Severe ENL reaction

-appearance of ENL nodules with ulceration

or

-ENL skin lesions with one or more of the following:

- loss of muscle strength and/or loss of sensation in hands, feet or eyes (reduction in VMT and/or ST): severe neuritis;

- painful eyes, with redness around the limbus cornea, increased lacrimation, constriction of the pupil and diminished vision (irido-cyclitis);

- enlarged tender lymphnodes (lymphadenitis)

- painful testicular swelling (orchitis)

- painful swollen joints (arthritis)

- painful swollen fingers (dactylitis); and

- general condition: severe fever and malaise.

Figure 1. Signs of reaction (adapted from ALERT's Manual for Field Treatment of Leprosy Reactions ${ }^{2}$ ).

New or increased nerve dysf unction is, for the sake of convenience, hereafter referred to as neuritis, although in some cases, which we may not be able to distinguish, deterioration of function may possibly be due to (post-reaction) scar formation in the nerves. Signs of neuritis and criteria for mild and severe neuritis are summarized in Figure 2.

\section{CLINICAL MANIFESTATIONS OF REACTION}

To facilitate a descriptive monitoring of what is seen in events, we also distinguish between the following elements of reactions:

where apparently no nerve involvement occurred; these are termed either: 'RR' for RR with skin reaction only, or 'ENL' for ENL reaction without neuritis;

conditions where nerve tenderness and/or nerve dysfunction are found without signs of reaction in the skin, termed: 'neuritis' or 'neuritis alone'; and

combinations: 'RR-with-neuritis', 'ENL-with-neuritis' and '( RR + ENL)-with-neuritis'.

The terms 'RR' and 'ENL' are normally used for the whole complex of the reactional process concerned, encompassing the pathology of skin, nerves and other organs. In this 
New (i.e., in existence for less than 6 months) nerve involvement in a leprosy patient is recorded as a case of: Mild neuritis

if only

- new or increased thickening of nerves

and/or

— paraesthesia, 'tingling'

is found, without pain or tenderness and without loss of function,

and as

Severe neuritis

if one or more of the following signs is found:

- tenderness of one (or more) nerve(s), with or without loss of nerve function

- new (or unquestionable increase of) sensory loss in the skin of palms and/or soles, i.e.:

2 or more new points of no response out of

4 points tested for the ulnar nerve

6 points tested for the median nerve

10 points tested for the tibial posterior nerve

by a standardised Sensory Test 'ST' carried out with a nylon filament producing a stimulus of $10 \mathrm{~g}$.

- new or increased muscle weakness: a decrease of muscle strength observed in a simplified 'Voluntary Muscle strength Test' (VMT), which distinguishes three grades only: S (for strong), W (for weak) and P (for paralysed). The VMT is done for the following nerves and functions:

facial nerve-tight closure of eye lids

ulnar nerve-abduction of 5th finger

median nerve-abduction of thumb

peroneal nerve-dorsiflexion of foot

radial nerve-dorsiflexion of hand

Figure 2. Signs of neuritis (adapted from ALERT's Manual for Field Treatment of Leprosy Reactions ${ }^{2}$ ).

field study we wish to describe the reactional phenomena in some detail. We therefore categorized the reactions, by clinical criteria (listed in Figures 1 and 2), into those where apparently only the skin was affected, others where only the nerves seemed to be affected and those with both skin and nerve involvement.

When an event is noticed, the LCS does a full review examination, scores the presence or absence of relevant symptoms and signs on the 'Prednisolone Treatment \& Report of Event Form' (PTREF), followed by a summary entry, just as for a periodical review, on the Patient Record Card.

At the monthly review of the cards (or, if he happens to be at the clinic, immediately on the spot) one of the MOs categorizes the event and indicates the presence of RR, ENL, or neuritis or combinations of these three categories on the PTREF.

\section{TREATMENT OF REACTION}

In most events prednisolone is prescribed for the patient. This treatment was for study patients the same as for ALERT's routine services. ${ }^{3} \mathrm{MB}$ patients receive a prednisolone course of 20 weeks. This course starts with $40 \mathrm{mg}$ daily for 2 weeks. The daily dose is then reduced to $30 \mathrm{mg}$, given for 4 weeks and then tapered down every 4 weeks, via $20 \mathrm{mg}$, $15 \mathrm{mg}, 10 \mathrm{mg}$, each for 4 weeks, and finally $5 \mathrm{mg}$ daily for the last 2 weeks. For PB patients the dosages of the prednisolone regimen are the same, but the daily dose is reduced every 2 weeks and the course thus lasts 12 weeks only. For pregnant women and for children shorter courses and lower dosages are prescribed. 
In case of no response or of a worsening of the reaction or the neuritis, the LCS is to increase the dose. The prednisolone course is then prolonged and may last some 6-12 or more weeks longer. ALERT's Manual ${ }^{2}$ details which conditions LCSs are allowed to handle themselves and in which conditions patients have to be referred to the hospital.

At the end of a corticosteroid course another full review is done and findings are recorded both on the PTREF and on the Patient Record Card. The data reported here are obtained from these two records.

\section{ANALYSIS}

Incidence rates for events have been calculated using a person-years-at-risk (pyar) approach. Since the examination at the start of MDT includes detailed enquiries into leprosy-associated findings during the preceding 6-month period, and reaction or neuritis of up to 6-months' duration is still counted and treated as an event, all patients were assumed to have been under 'observation' for this period. There followed a period of MDT, and, after appropriate completion of MDT, a period of surveillance. Patients who had had a 6-monthly review within 6 months of the time limit of this analysis (30 June 1992) were assumed to be on treatment or surveillance up to this date. Patients who had no review after the end of 1991 were assumed to have been observed for 3 more months after their last review.

Statistical comparisons of numeric quantities have been made calculating $95 \%$ confidence intervals (CIs) of differences between means. Thus a statistical difference exists when the $95 \% \mathrm{CI}$ excludes zero.

\section{Patients}

We report on 286 patients, $128 \mathrm{~PB}$ and $158 \mathrm{MB}$, enrolled in the first 3 years of the project, from April 1988 onwards. Procedures and results of patient enrolment, completion of MDT treatment and case holding were described in Part $\mathrm{I} .{ }^{1}$ Observations up to the end of June 1992 covered 267.0 person-years among PB patients (mean $2 \cdot 1$ years per patient). Similarly for MB patients, observations covered $402 \cdot 1$ person-years (mean $2 \cdot 5$ years).

\section{Results}

EVENTS IN PB PATIENTS

Of the 128 PB patients, $27(21 \cdot 1 \%)$ experienced a total of 35 events. All events occurred in patients classified as BT. This corresponds to an incidence rate of 131 per 1000 (pyar). The 35 events were distributed among the 128 patients with 101 patients having no event, 23 patients having 1 event, 2 having 2, 1 having 3 and 1 patient having 5 events. This differed significantly from a random (Poisson) distribution $\left(\chi^{2}=667,5 \mathrm{df}\right)$. Of the first observed reaction for each patient, 14 out of $27(51 \cdot 9 \%)$ showed RR with neuritis, 11 $(40 \cdot 7 \%)$ neuritis alone, and 2 had R R alone.

The clinical manifestations and the chronology of events in relation to treatment and surveillance are shown in Figure 3. Neuritis was present in 33 of the 35 events. Two events were observed with RR skin reaction alone, i.e. with, by our method of detection, 


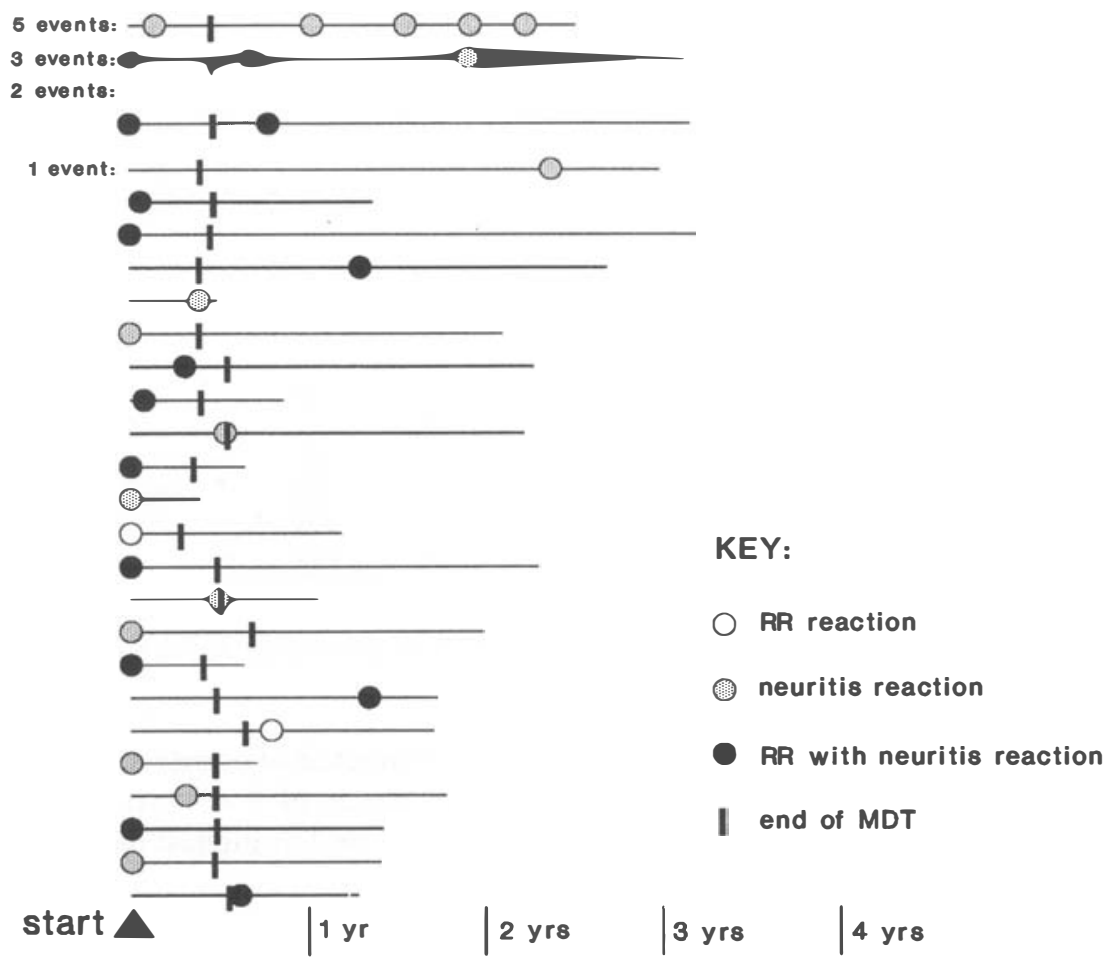

Figure 3. Clinical manif estations and chronology of 35 events observed in 27 out of 128 PB patients in the AMFES project, up to the end of June 1992. Patients with an 'end of MDT' mark less than 6 months from the start of MDT underwent an early end-of-treatment assessment, followed by unsupervised completion of MDT, for reasons of inaccessibility during the rainy season.

no evidence of neuritis. In 15 of the 17 events with neuritis alone, the neuritis was 'silent': the new or increased nerve dysfunction occurred not only without accompanying signs of reaction in the skin, but also without any pain or nerve tenderness. ${ }^{4,5}$

The 3 patients with 2 or 3 events had RR-with-neuritis in their first reactions, but 2 had neuritis alone in later events. The woman in whom 5 events were observed had neuritis alone on each occasion. She started with spells of silent neuritis, but by the 5th event she had several tender nerve trunks.

In $13(48 \cdot 1 \%)$ of the 27 patients who had events, a reaction was found at the time of diagnosis and start of MDT. Another $9(33 \cdot 3 \%)$ had an event during the treatment course and 5 patients had their first event after MDT. A total of $13(37 \cdot 1 \%)$ of the 35 events were observed after MDT, over 139.5 pyar during surveillance. Thus the incidence of events during surveillance after MDT was 93 per 1000 pyar. Of these, 4 reactions occurred in the first 6 months after MDT, 3 in the second half year, 3 in the third, 2 in the fourth half year, and 1 in the fourth year after MDT (and this was apparently* not a relapse). Separate incidence rates of events, before and during MDT, and for 2 periods after MDT are shown in Figure 4.

\footnotetext{
* Only some of the previously existing skin lesions had become red and raised again. On a course of prednisolone, without any antileprosy treatment, these lesions subsided nicely. The patient remains under surveillance.
} 


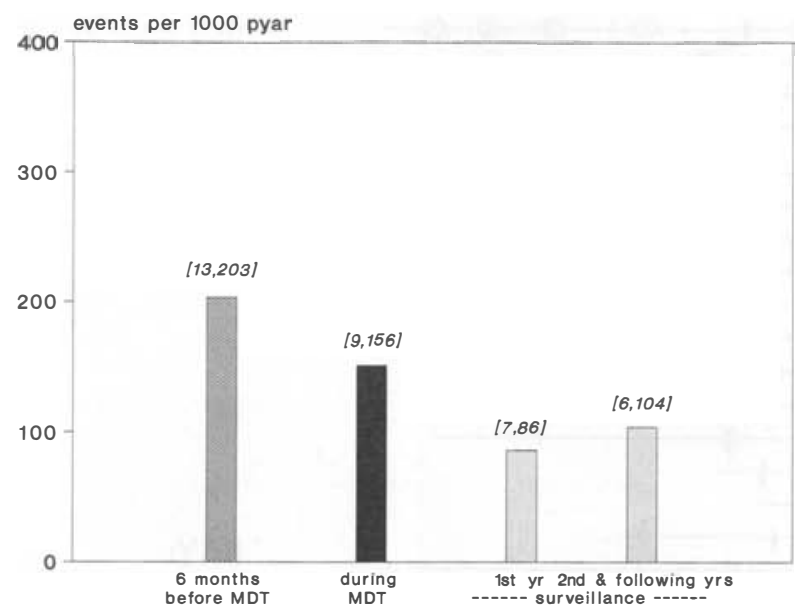

Figure 4. Incidence of 35 events in $128 \mathrm{~PB}$ patients in the AMFES project before, during and after treatment.

The relation between disability at start and the occurrence of events in PB patients is shown in Table 1 . The 58 patients with a disability grade of 0 at start of MDT had significantly less events than the 70 patients with either 1 or 2 as highest disability grade. $\left(\chi^{2}=6 \cdot 231 \mathrm{df}, p=0 \cdot 013\right)$.

Also the mean score of HF-impairment (the sum of the disability grades of a patient's 4 extremities) ${ }^{1}$ at the start of MDT among the 16 patients who subsequently experienced events was 2.63 (sd 2.47), compared with the remaining 112 patients for whom that mean score was $1 \cdot 75$ (sd $2 \cdot 22)(95 \%$ CI of difference $-0 \cdot 3$ to $2 \cdot 1$, not significant). However, PB patients who already had some impairment at the start of MDT were 2.5 times more likely to experience further events than those without existing impairments (relative risk $2 \cdot 5,95 \%$ CI $0 \cdot 9$ to $7 \cdot 3$ ).

For an assessment of changes in the HF-impairment scores of patients over time we have compared scores at start of MDT with latest recorded scores, calculating the change in score for each patient. Of 106 PB patients for whom valid scores were

Table 1. Occurrence of events in 27 patients who experienced 1 or more events, out of 128 $\mathrm{PB}$ patients in the AMFES project, in relation to their disability grades at the start of MDT

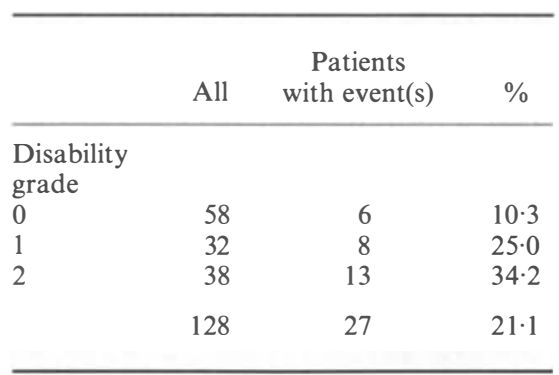




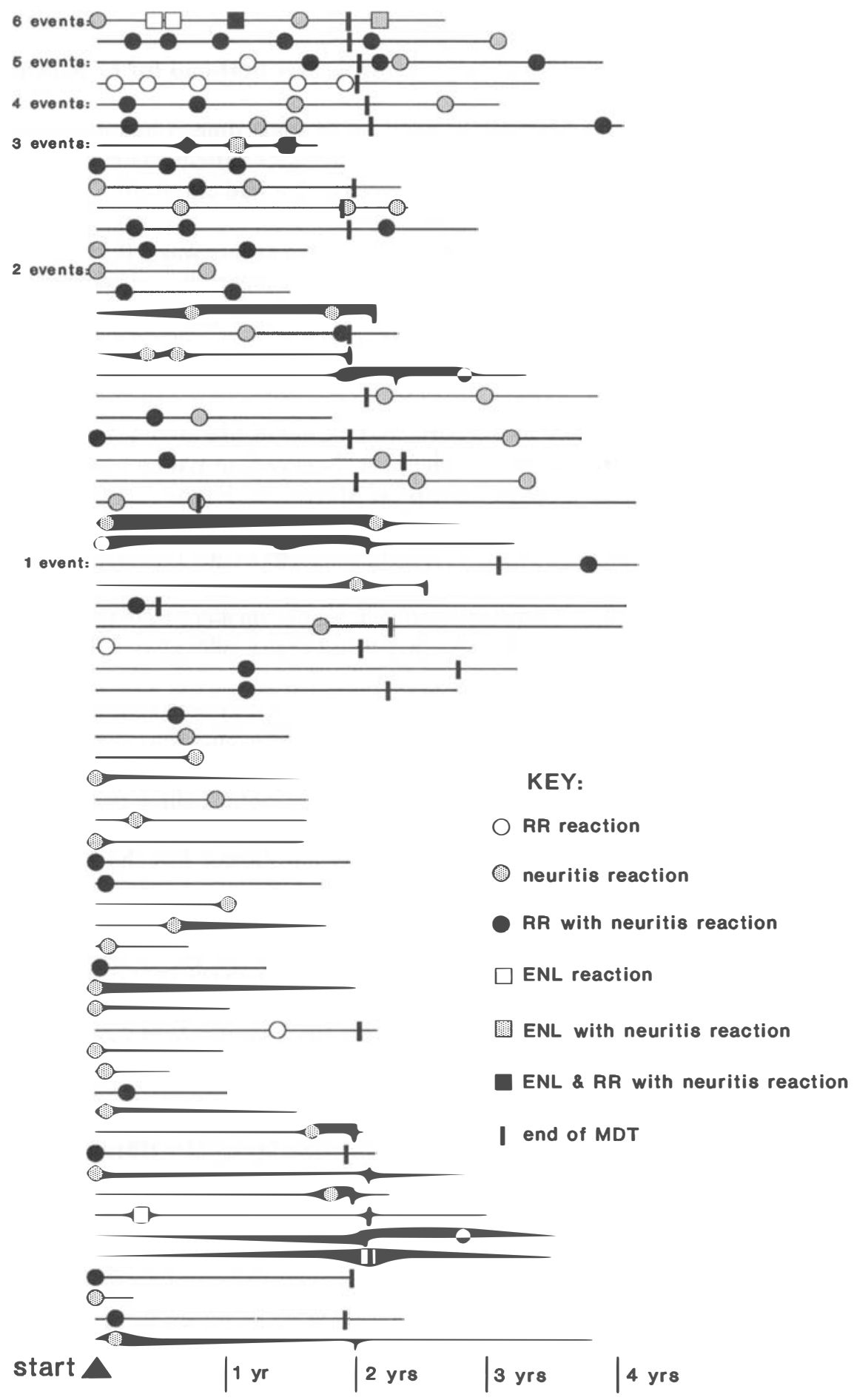

Figure 5. Clinical manifestations and chronology of 114 events observed in 64 out of $158 \mathrm{MB}$ patients in the AMFES project, up to the end of June 1992. The 2 cases where 'end of MDT' occurred less than 1 year after the start concerned two skin-smear negative BT patients who were taken off MB-MDT early. 
available, 27 had improved, 12 had become worse over time and for 67 patients initial and final scores were the same.

Among 26 patients who experienced an event at some time, 5 had improved, 9 had become worse and 12 ended with no change in score (1/27 patients with an event had no final score). Among 80 patients with both scores who had no event, 22 improved, 3 became worse and 55 ended with the same score.

The mean change in scores for the 26 patients with events was $-0 \cdot 31$ (sd 1.12), and for the 80 without events $0 \cdot 49$ (sd 1.11). The difference in mean change between these two groups was $0.80(95 \% \mathrm{CI}$ of difference 0.3 to $1 \cdot 3)$ which is significant.

\section{EVENTS IN MB PATIENTS}

Of the $158 \mathrm{MB}$ patients, $64(40 \cdot 5 \%)$ experienced a total of 114 events. This corresponds to an overall incidence rate of 284 per 1000 pyar. Ninety-four patients had no event, 38 had 1 event, and 26 had multiple events as shown in Figure 5. This also differs significantly from a Poisson distribution $\left(\chi^{2}=296 \cdot 7,6 \mathrm{df}\right)$.

The majority of events $(82 / 114,71.9 \%)$ were observed in $(46 / 101)$ BL patients during $248 \cdot 3$ pyar, corresponding to an incidence for BL patients of 330 events per 1000 pyar. Six events occurred in (4/9) BT patients during 26.6 pyar, incidence 226 per 1000 pyar, 24 in (13/47) LL patients during 124.4 pyar, incidence 193 per 1000 pyar, and two events in the single NL patient.

The clinical manifestations and the chronology of all events in relation to treatment and surveillance are shown in Figure 5. Sixteen of the 114 events were present at the time of diagnosis and start of MDT, 79 events occurred during treatment, and so far 19 events have been seen during surveillance following MDT. Amongst the 6 patients with 4 or more events was one (male BL patient) for whom all events consisted of a pronounced RR skin reaction without any evidence of any nerve problems. For the 5 other patients the manifestations of reaction differed over time.

The clinical manifestations of the first events of MB patients are listed in Table 2. Neuritis was also the most common element of reaction in the MB patients. In these first events it was seen in $56(87.5 \%)$ of the 64 patients, and in $33 / 64(51.6 \%)$ there were not any signs of reaction in the skin. Of the events consisting of neuritis alone, $35 / 53(66.0 \%)$ were cases of 'silent neuritis' in which there was no tenderness in any of the palpated nerves.

Table 2. Clinical manif estations of first reactions among 64 out of $158 \mathrm{MB}$ patients in the AMFES project who experienced 1 or more reactions

\begin{tabular}{lcccc}
\hline $\begin{array}{l}\text { Components } \\
\text { of reaction: }\end{array}$ & RR & Neuritis & ENL & Total events \\
\hline $\begin{array}{l}\text { Reaction type } \\
\text { RR (skin alone) }\end{array}$ & 6 & - & - & 6 \\
$\quad$ Neuritis alone & - & 33 & - & 33 \\
ENL (skin alone) & - & - & - & 23 \\
RR-with-neuritis & 23 & 23 & 2 & 64 \\
Total events & 29 & 56 & & \\
\hline
\end{tabular}




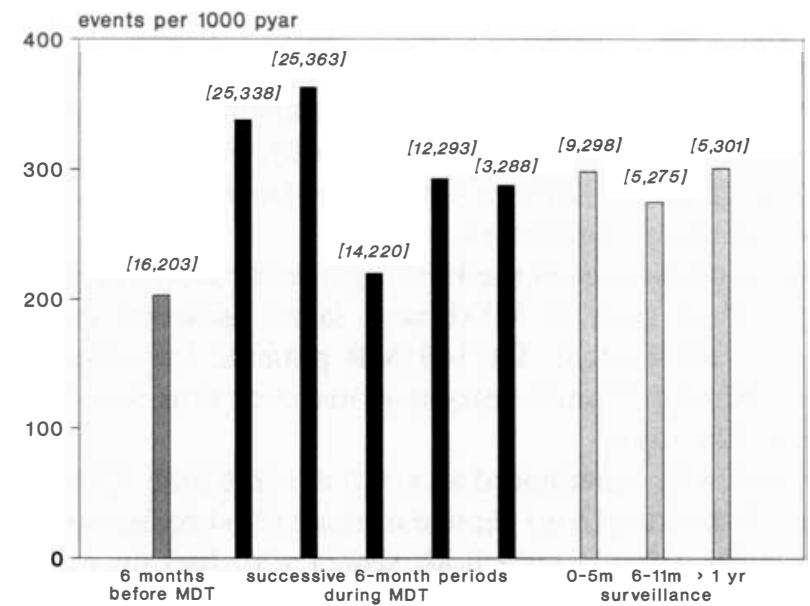

Figure 6. Incidence of 114 events in $158 \mathrm{MB}$ patients in the AMFES project before, during and after treatment.

Of the 64 patients with events, $16(25.0 \%)$ already had their first event at the start of treatment, $32(50 \cdot 0 \%)$ experienced events in the 1 st year of treatment, $12(18.8 \%)$ in the 2nd or 3rd year of treatment and 4 patients $(6.3 \%)$ had their first event during surveillance (Figure 5).

During the first year of treatment, 50 events occurred during 142.9 pyar, giving an incidence of 350 per 1000 pyar. The 29 events recorded during treatment after the first year related to $115 \cdot 1$ pyar, an incidence of 252 per 1000 pyar. The 19 events during surveillance were recorded in the 64 patients who had so far completed MDT, over 65.0 pyar in surveillance. This corresponds to an incidence rate of 292 per 1000 pyar. In Figure 6 incidence rates are given per period of 6 months from before treatment until 1 year after MDT, and thereafter in 1 bar for patients who were on surveillance for more than 1 year.

The relation between disability at start and the occurrence of events in MB patients is shown in Table 3. The 68 patients with a disability grade of 0 at start of MDT had a similar occurrence of events to the 90 patients with either 1 or 2 as highest disability grade.

Table 3. Occurrence of events in 64 patients who experienced 1 or more events, out of $158 \mathrm{MB}$ patients in the AMFES project, in relation to their disability grades at the start of MDT

\begin{tabular}{|c|c|c|c|}
\hline & All & $\begin{array}{c}\text { Patients } \\
\text { with event(s) }\end{array}$ & $\%$ \\
\hline \multicolumn{4}{|c|}{$\begin{array}{l}\text { Disability } \\
\text { grade }\end{array}$} \\
\hline 0 & 68 & 26 & $38 \cdot 2$ \\
\hline 1 & 52 & 28 & $53 \cdot 8$ \\
\hline \multirow[t]{2}{*}{2} & 38 & 10 & $26 \cdot 3$ \\
\hline & 158 & 64 & $40 \cdot 5$ \\
\hline
\end{tabular}


Also the scores of HF-impairment at the start of MDT of the 54 patients who subsequently experienced events (mean of scores $1 \cdot 50$, sd 1.6), were not significantly different from the scores of the remaining 104 patients (mean of scores 1.97, sd 2.2) (95\% CI of difference $-0 \cdot 2$ to $1 \cdot 1)$. MB patients who already had some impairment at the start of MDT were thus equally likely to experience further events as patients starting treatment without existing impairments.

For an assessment of changes in the HF-impairment scores of patients over time, we have compared scores at start of MDT with latest recorded scores, calculating the change in score for each patient. Of $140 \mathrm{MB}$ patients for whom valid scores were available, 35 had improved, 25 had become worse over time and for 80 patients initial and final scores were the same.

Among 62 patients who experienced an event at some time, 15 had improved, 23 had become worse and 24 ended with no change in score (2/64 patients with an event had no final score). Among 78 patients with both scores who had no event, 20 improved, 2 became worse and 56 ended with the same score.

The mean change in scores for the 62 patients with events was $-0 \cdot 15$ (sd 1.47), and for the 78 without events 0.47 (sd 0.95). The difference in mean change between these two groups was $0.62(95 \%$ CI of difference 0.2 to $1 \cdot 0)$ which is significant.

\section{Discussion}

In Hastings' handbook ${ }^{4}$ leprosy reaction is described as 'the appearance of symptoms and signs of acute inflammation in lesions of a patient with leprosy. Clinically there is redness, swelling and sometimes tenderness of skin lesions, and swelling, pain and tenderness of nerves, often accompanied by loss of function. New lesions may appear'. Thus it is not made unequivocally clear that a leprosy reaction may appear as an episode of neuritis alone, without clinical signs of reaction in the skin. Furthermore, the quoted description seems to exclude cases of silent neuritis. ${ }^{5,6}$ From a further description in the same chapter, and also elsewhere in the literature ${ }^{7,8}$ it is evident that neuritis alone, including silent neuritis, is presently recognized as a possible manifestation of a leprosy reaction. This evident lack of a succinct and comprehensive definition of a leprosy reaction remains a problem.

In this field study project we have recorded and treated new or increased nerve dysf unction as 'neuritis', although we are aware that in some cases the loss of nerve function may be due either to postreaction scar formation ${ }^{9,10}$ in a nerve or, particularly in untreated progressive disease, to pathological processes other than those associated with RR or ENL reaction. ${ }^{7}$

There have been very few studies of leprosy reactions on a longitudinal basis. In the only other prospective study in Africa we are aware of, in Malawii, ${ }^{11}$ PB patients starting WHO-MDT at a much earlier stage of disease (many of them detected in active case finding) experienced much less reversal reaction. At the start of treatment, 11 of 503 patients $(2 \cdot 2 \%)$ had a reaction compared to $13 / 128(10 \cdot 2 \%)$ in AMFES. During treatment 7 reactions among 488 Malawi patients gave an incidence rate of 28/1000 pyar, compared to an incidence of 156 events per 1000 pyar in this AMFES group. In the first year of surveillance of PB patients, the Malawi istudy observed 15 reactions among 314 patients (who had been self-reporting for start of MDT) giving an incidence rate of 
48/1000 pyar (AMFES: 88/1000 pyar). In further follow up for 3 more years involving 471 patients, only 2 reactions were seen (no incidence rate given) ${ }^{12}$ and among our 102 patients we have observed already 6 reactions (incidence rate 99/1000) in years 2, 3 and 4 of surveillance.

Whilst for all periods the incidence of reaction is higher among the Ethiopian patients, the findings in both studies indicate that for these PB patients the incidence of reaction during the first year of surveillance is not very different from the incidence during the MDT course. This finding, combined with our finding of a higher risk of subsequent reaction in PB patients with an existing nerve function impairment at the start of MDT, may thus be taken as an indication for (more) surveillance attention during that first year, particularly for patients who already have some nerve function impairment. Whether or not this tentative conclusion is supported by further findings in this project, our deduction from these preliminary findings may illustrate the potential of this type of study.

In our study data, both reaction incidence rates and proportions of patients with reactions showed an approximate 2:1 ratio between $\mathrm{MB}$ and $\mathrm{PB}$ patients. However, we suggest that reaction incidence rates, as also reported by Boerrigter et al. ${ }^{1,12}$ (and quoted in previous paragraphs), are a more meaningful measure.

Our results are comparable to the findings of a contemporary retrospective study on records of ALERT's field services, ${ }^{13}$ which also concluded that in the MB category approximately twice as many patients had reactions as in the PB group. However, our finding of over $10 \%$ of patients in reaction at their initial presentation is higher than the $3 \cdot 5 \%$ reported by Becx-Bleumink and Debrezion Behre. ${ }^{13}$ As both studies concerned very similar patients, in virtually identical services, this discrepancy may reflect a difference between our prospective study and their analysis of retrospective data.

In both $\mathrm{MB}$ and $\mathrm{PB}$ groups, reactions were not randomly distributed among patients. Whilst this may in part be due to our definition of a 'new' reaction, it is nevertheless clear that certain patients experienced long periods containing either repeated 'new' reactions or prolonged episodes of on-going reaction activity. Clearly therefore the definition of a reaction also influences incidence rates.

Because of the nonindependence of multiple reactions in individual patients, we have considered clinical manifestations of the first event only for each patient. Over $85 \%$ of first reactions included neuritis, of which approximately half did not involve the skin and most of the latter were episodes of silent deterioration of nerves, without any pain or tenderness. Thus, without caref ul surveillance of nerve function, a large proportion of the occurrence of new or additional nerve dysfunction would not have been detected.

The incidence of events among PB patients was much lower in general than in MB patients, but in both groups similar incidence rates were found during treatment and surveillance periods. Whilst in PB patients pre-existing disability at the start of treatment was a significant risk factor for a subsequent event, this was not found for the $\mathrm{MB}$ patients.

Over all patients, the mean change in HF-impairment score between the start of treatment and the latest score represented a deterioration among patients who experienced events, but an improvement among those without events. This difference was statistically significant. Whilst in itself this finding is unremarkable, in view of the common factors in the definition of an event and the HF-impairment score, it supports the validity of the HF-impairment score concept, and of considering mean longitudinal changes in the score when comparing different groups of patients. 


\section{Acknowledgments}

We gratef ully acknowledge the good daily care given to the patients and the many records taken by the leprosy control supervisors and health assistants.

The project is financed by ILEP as a joint project of various organisations coordinated by NSL, the Netherlands Leprosy Relief Association.

\section{References}

1 Rijk AJ de, Shibru Gabre, Byass P, Theodroes Berhanu. Field evaluation of WHO-MDT of fixed duration, at ALERT, Ethiopia: the AMFES project-I: MDT course completion, case holding and a nother score for disability grading. Lepr Rev, 1994; 65: 305-319.

2 All Africa Leprosy \& Rehabilitation Centre, ALERT. Manual for field treatment of leprosy reactions. 2nd rev. ed. Addis Ababa: ALERT, 1989.

${ }^{3}$ Becx-Bleumink M, Berhe D, Mannetje W 't. The management of nerve damage in the leprosy control services. Editorial, Lepr Rev, 1990; 61: 1-11.

${ }^{4}$ Pfaltzgraff RE, Bryceson A. Clinical leprosy. In: Leprosy, Hastings RC (ed). London: Churchill Livingstone, 1985, 165-71.

5 Duncan ME, Pearson JMH. Neuritis in pregnancy and lactation. Int J Lepr, 1982; 50: 31-8.

${ }^{6}$ Hamilton J. Deformity prevention in the field: a systematic approach. Lepr Rev, 1983; 54: 229-37

7 Pearson JMH, Ross WF. Nerve involvement in leprosy: pathology, differential diagnosis and principles of management. Lepr Rev, 1975; 46: 199-212.

${ }^{8}$ Rose P, Waters MFR. Reversal reactions in leprosy and their management. Editorial, Lepr Rev, 1991; 62: $113-21$.

9 Sunderland S. The internal anatomy of nerve trunks in relation to the neural lesions of leprosy; observations on pathology, symptomatology and treatment. Brain, 1973; 96: 865-88.

${ }^{10}$ Charosky CB, Gatti JC, Cardama JE. Neuropathies in Hansen's disease. Int J Lepr, 1983; 51: 576-86.

11 Boerrigter G, Ponnighaus JM, Fine PE. Preliminary appraisal of a WHO- recommended multiple drug regimen in paucibacillary leprosy in Malawi. Int J Le pr, 1988; 56: 408-17.

12 Boerrigter G, Ponnighaus JM, Fine PEM, Wilson RJ. Four-year follow-up results of a WHO-recommended multiple drug regimen in paucibacillary leprosy patients in Malawi. Int J Lepr, 1991; 59: 255-61.

13 Becx-Bleumink M, Debrezion Berhe. Occurrence of reactions, their diagnosis and management in leprosy patients treated with multidrug therapy. Experience in the leprosy control program of the All Africa Leprosy and Rehabilitation Center (ALERT) in Ethiopia. Int J Lepr, 1992; 60: 173-84. 